\title{
Un nouveau genre de Trematoda Digenea, parasite de poissons marins d'Ensenada, Baja California, (Mexique. II)
}

\author{
par Eduardo CABALLERo y C. * et Guillermina CABALLERO R. ** \\ * Instituto de Biología, U.N.A.M. Apartado postal n 692. México 1, D.F., México \\ ** Laboratorio de Helmintología del Instituto de Biología, U.N.A.M. \\ Apartado postal 70-153 México 20, D.F., México
}

\section{Résumé.}

Un exemplaire d'un trématode récolté chez le poisson marin Paralichthys woolmani Jordan et William, 1896, de la Péninsule de Baja California, Mexique, est décrit et discuté. Nous pensons qu'il appartient à un nouveau genre et à une nouvelle espèce caractérisé par la présence d'une très grande poche du cirre, située sur l'axe médian du corps, d'une glande prostatique petite, d'un cirre court et cylindrique et d'un ovaire multilobé au centre du corps.

\section{Summary.}

A new genus of Trematoda Digenea that parasitizes marine fishes from Ensenada, Baja California, México. II.

A trematode collected from the marine fish Paralichthys woolomani Jordan and William, 1896, from the Baja California, Peninsule, Mexico, is described and discussed. It belongs to a new genus and to a new species because of the presence of a big cirrus pouche, located in the middle line of the body; of a little prostatique gland; of a small and cylindrical cirrus and finally, a multilobed ovary in the central region of the body.

L'unique exemplaire mûr d'après lequel la description a été réalisée correspond au genre type. Cet exemplaire mûr, plus deux autres immatures, ont été récoltés dans l'intestin de Paralichthys woolmani, le 29 novembre 1967, à Ensenada, Baja California, Mexique. Ces parasites ont été fixés au Bouin, colorés par la méthode trichromique de Gomori, éclaircis au Salycilate de méthyle et montés au Baume du Canada. 
Dermaskrjabinia macrobursa. gen. nov., spéc. nov.

Le parasite petit, rond et épais (fig. 1) mesure 1,632 $\mathrm{mm}$ de diamètre antéropostérieur sur $1,760 \mathrm{~mm}$ de diamètre transversal. La cuticule inerme recouvre un tégument épais formé de deux couches bien différenciées: une externe de fibres transversales

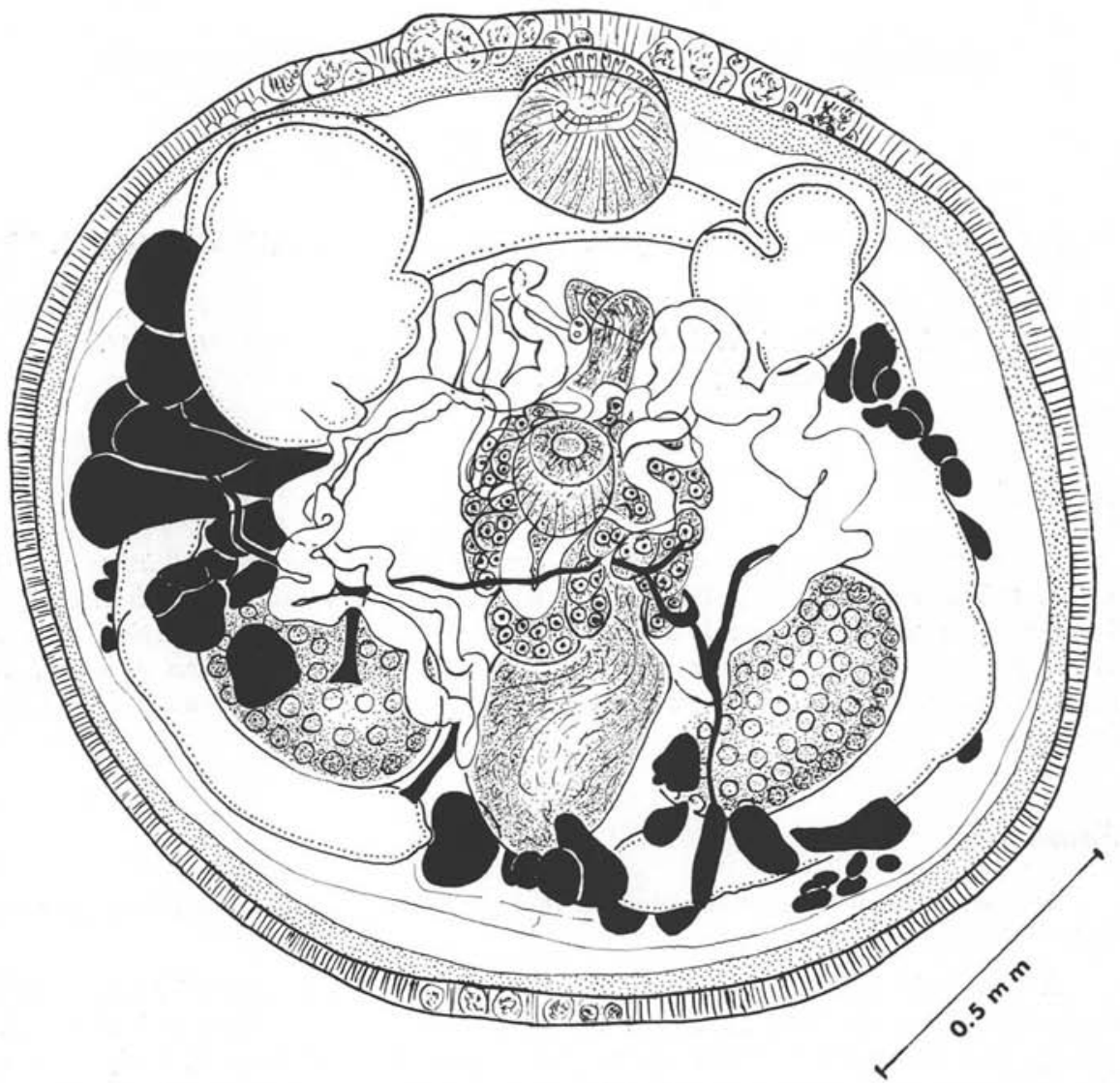

FIg. 1. - Dermaskrjabinia macrobursa gen. nov., spec. nov. Preparation in toto. Vue ventrale. Holotype

et une interne granuleuse dont l'épaisseur est de $0,043 \mathrm{~mm}$ et $0,039 \mathrm{~mm}$ respectivement. Dans la partie antérieure du corps en avant de la ventouse buccale et en moindre quantité dans la partie postérieure du corps, s'observent dans la couche externe tégumentaire des masses ovoïdes d'aspect granuleux probablement d'origine glandulaire. 
La ventouse buccale subterminale, sphérique et fortement musculeuse, présente dans son bord antérieur une structure semilunaire projetée vers le bord externe de la couche tégumentaire interne; la portion antérieure de cette structure présente une rangée de corps bifides et mesure $0,224 \mathrm{~mm}$ de diamètre antéropostérieur sur $0,279 \mathrm{~mm}$ de diamètre transversal. L'acétabulum est plus petit que la ventouse buccale, ovale, situé au niveau de la partie antérieure de l'ovaire et sur l'axe mi-ventral, il est aussi très musculeux et son ouverture forme un anneau à muscles radiaux, il mesure $0,185 \mathrm{~mm}$ de diamètre antéropostérieur sur $0,159 \mathrm{~mm}$ de diamètre transversal. Le rapport ventouse buccale/acétabulum est : $1: 1,2 \times$ 1: 1,7 .

La bouche se trouve dans la partie antéroventrale de la ventouse buccale, elle possède un anneau de muscles circulaires et une lèvre également circulaire portant des papilles. Le pharynx n'a pas été observé. Les caecums larges s'étendent latéralement jusqu'à la région postérieure du corps; les portions antérolatérales de ces caecums forment de grandes poches, lesquelles sont, peutêtre, le résultat de variations osmotiques.

Les pores reproducteurs, situés entre les deux ventouses, se trouvent à $0,480 \mathrm{~mm}$ du bord antérieur du corps. Les testicules sont situés dans la moitié postérieure du corps, de chaque côté de la ligne médiane; de forme ovoïde, grands, à bords lisses, ils mesurent, à droite, 0,236 $\mathrm{mm}$ de long sur 0,236 $\mathrm{mm}$ de large et, à gauche, $0,344 \mathrm{~mm}$ de long sur $0,279 \mathrm{~mm}$ de large. La poche du cirre (fig. 2) est l'organe carac-

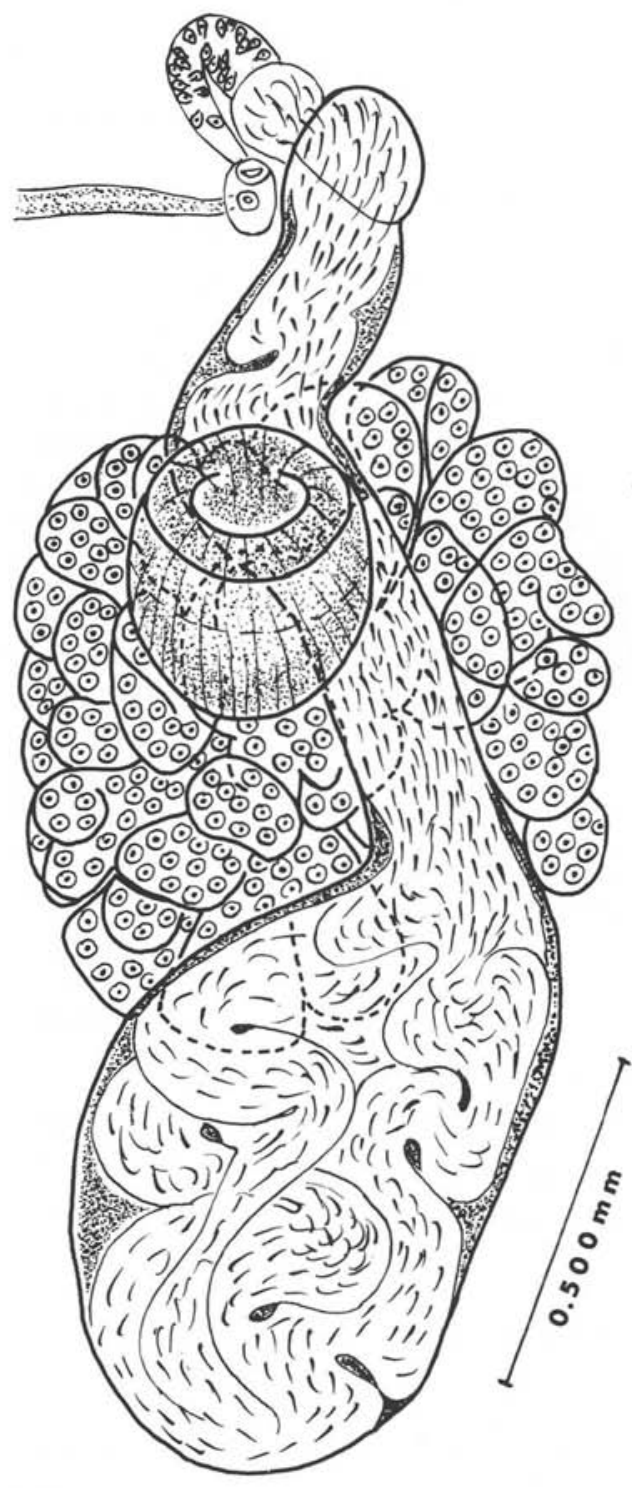

FIG. 2. - Dermaskrjabinia macrobursa gen. nov., spec. nov. Détail de la poche du cirre et des pores génitaux. Holotype. 
téristique de ce genre, elle est cylindrique en forme de bouteille à long col ; elle s'étend au niveau des follicules vitellogènes caudaux sur la ligne médiane du corps, donc en avant la terminaison des caecums jusqu'aux pores reproducteurs ; elle mesure $1,118 \mathrm{~mm}$ de longueur sur $0,172 \mathrm{~mm}$ de largeur. La vésicule séminale occupe presque toute la poche du cirre, elle est très sinueuse, ce qui lui donne l'apparence d'être divisée; elle mesure $1,010 \mathrm{~mm}$ de long sur $0,107 \mathrm{~mm}$ de large; la glande prostatique occupe la portion terminale de la poche du cirre; le cirre est inerme, cylindrique et court.

L'ovaire ventral, occupe la région centrale du corps, il est multilobé, plus étendu à droite qu'à gauche, et possède une petite cavité postérieure occupée par la glande de Mehlis et l'ootype; il mesure $0,361 \mathrm{~mm}$ de long sur 0,387 $\mathrm{mm}$ de large. L'utérus se trouve dans l'espace interne du corps, les anses ascendantes, des-

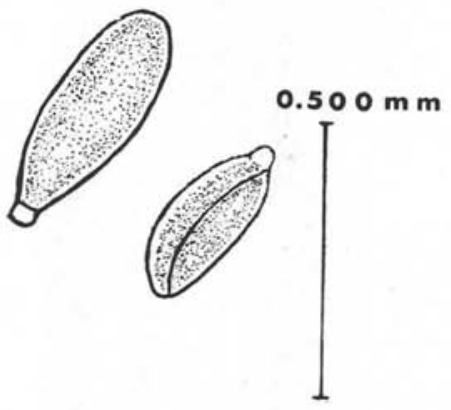

FIG. 3. - Dermaskrjabinia macrobursa gen. nov., spec. nov. Eufs. Holotype. cendantes et transversales sont pleines d'œufs operculés, mamelonnés (fig. 3). Les œufs mesurent $0,026 \mathrm{~mm}$ de long sur $0,013 \mathrm{~mm}$ de large.

Les glandes vitellogènes sont formées de grands follicules ovoïdes, s'étendant dans les champs latéraux et postérieur du corps, du niveau de la bifurcation intestinale jusqu'en arrière de la poche du cirre; quelques follicules mesurent de 0,107 à $0,133 \mathrm{~mm}$ de long sur 0,112 à $0,353 \mathrm{~mm}$ de large. Les vitelloductes transversaux aboutissent dans la région de l'ootype, ils sont en communication avec deux autres conduits longitudinaux.

Le pore excréteur n'a pas été observé.

Hôte : Paralichthys woolmani, Jordan et William, 1896. Pisces : Paralichthyidae.

LOCALISATION : Intestin.

Localité: Ensenada, Baja California, Mexique.

Holotype: Collection helminthologique de l'Institut de Biologie de l'Université Nationale Autonome du Mexique, $\mathrm{n}^{\circ}$ 226-15.

\section{Dermaskrjabinia. gen. nov.}

Définition: Lepocreadiidae. Corps petit, rond, à cuticule nue ; tégument épais, constitué de deux couches: une externe fibreuse longitudinale et une autre interne granuleuse. Ventouse buccale subterminale, grande, sphérique, musculeuse, portant une structure semilunaire pourvue de barres bifides. Acétabulum plus petit que la ventouse buccale, musculeux, situé dans la région médio-ventrale du corps, pourvu d'un anneau de muscles radiaux. Bouche antéroventrale pourvue d'une lèvre papilliforme. Pharynx absent. Caecums larges, latéraux, s'étendant jusqu'au bord postérieur 
du corps. Pores reproducteurs à mi-distance entre les deux ventouses. Testicules postérolatéraux. Poche du cirre très grande, occupant l'axe médio-ventral du corps, du niveau des follicules vitellogènes plus postérieurs jusqu'au pore reproducteur. Vésicule séminale grosse, sinueuse, remplissant tout le fond de la poche jusqu'à la région antérieure. Glande prostatique petite, dans la région antérieure de la poche du cirre. Cirre court et cylindrique. Ovaire multilobé dans la région ventro-centrale du corps. Glande de Mehlis et ootype dans la région postérieure de l'ovaire. Utérus dans la région interne du corps. Eufs à coque lisse, operculés, avec un mamelon sur un des pôles. Follicules vitellogènes en petit nombre, grands et petits, occupant les aires latérales et postérieures, du niveau de la bifurcation intestinale jusqu'en arrière de la poche du cirre.

Espèce type: Dermaskrjabinia macrobursa gen. nov., spéc. nov.

Habitat: Parasite de l'intestin de poissons marins de la famille Paralichthyidae de Baja California, Mexique.

\section{Discussion}

Par la présence d'une grande poche du cirre et d'une grande vésicule séminale ainsi que par la forme et la structure de l'ovaire et des vitellogènes, on considère que ce parasite correspond à un genre nouveau qui se différencie des autres genres de la famille Lepocreadiidae.

Nous dédions Dermaskrjabinia macrobursa gen. nov., spéc. nov., en hommage posthume, au Maître éminent de l'Helminthologie mondiale, l'Académicien Konstantin Ivanovich Skrjabin.

\section{Bibliographie}

ARAi (H. P.), 1963. - Tremátodos digéneos de peces marinos de Baja California, México. An. Inst. Biol. Univ. Nac. Aut, México, 33 (1-2), 113-130.

Cable (R. M.) et Hunninen (A.) (V.), 1942. - Studies on Deropristis inflata (Molin), its life history and affinities to trematodes of the family Acanthocolpidae. Biol. Bull. 80. (2), 292-312.

Hafeezullah (M.), 1970. - Lepocreadid trematodes of marine fishes of India. Parasit., 61 (3), 345-356.

Howell (M.), 1966. - A new Genus in the Family Lepocreadiidae (Trematoda: Digenea) with Notes on the Status of some Taxa within the Family. Trans. Roy. Soc. New Zealand, 8, (3), 23-29.

Madhavi (R.), 1972. - Digenetic trematodes from marine fishes of Waltair coast, Bay of Bengal. I. Family Lepocreadiidae. Jour. Paras. 58 (2), 217-225.

Manter (H.W.), 1940. - Digenetic trematodes of fishes from the Galapagos Islands and the neighboring Pacific. Allan Hancock Pac. Exp. 2 (14), 329-497. 
—, 1963. - Studies on Digenetic Trematodes of Fishes of Fiji. II. Families Lepocreadiidae. Opistholebetidae, and Opecoelidae. Jour. Paras., 49 (1), 99-113.

- et van Cleave (H. J.), 1951. - Some digenetic trematodes, including eight new species from marine fishes of La Jolla, California. Proc. U.S. Nat. Mus., 101 (3279), 315-340.

Montgomery (W. R.), 1957. - Studies on digenetic trematodes from marine fishes of La Jolla, California. Trans. Am. Micr. Soc. 76 (1), 13-36.

SkrJabin (K. I.) et Koval (V. P.), 1960. - Nadsemeystvo Lepocreadioidea Cable, 1956. Trematody jivotnyjii cheloveka. Osnovy trematodologii. 18, 15-376. Isdatelstvo Akademii Nauk. S.S.S.R. Moskva.

Winter (H. A.), 1957. - Trematodos de peces marinos de aguas mexicanas. XIII. Cuatro digéneos de peces del océano Pacífico, dos de ellos, nuevas especies de la familia Cryptogonimidae Ciurea, 1933. An. Inst. Biol. Univ. Nac. Aut, México, 28 (1-2), 175-194.

—, 1960. - Algunos tremátodos digéneos de peces marinos de aguas del océano Pacífico del sur de California, U.S.A. y del litoral mexicano. An. Inst. Biol. Univ. Nac, Aut. México, 30 (1-2), 183-208.

Yamaguti (S.), 1958. - Systema Helminthum. The Digenetic Trematodes of Vertebrates. 1 (1-2): XI-1575. Interscience, Publ., New York, London.

-, 1971. - Synopsis of Digenetic Trematodes of Vertebrates. I et 2, 1-1074. Keigaku Publishing Co., Tokyo, Japan. 